\title{
Cellular imaging demonstrates genetic mosaicism in heterozygous carriers of an X-linked ciliopathy gene
}

\begin{abstract}
Sung Pyo Park ${ }^{1,2}$, In Hwan Hong ${ }^{2}$, Stephen H Tsang ${ }^{\star, 1,3}$ and Stanley Chang ${ }^{1}$
X-linked retinitis pigmentosa (XLRP) is the least common genetic type of retinitis pigmentosa; however, it has extremely devastating consequences to patients' activities of daily living. RPGR and $R P 2$ genes expressed in the photoreceptor sensory cilia are predominantly implicated in XLRP; however, the interpretation of genetic mutations and their correlation with clinical phenotypes remain unknown, and the role of these genes in photoreceptor cilia function is not completely elucidated. Therefore, we evaluated structural characteristics in five female obligate carriers of XLRP by using state-of-the-art non-invasive imaging methods, including adaptive optics (AO) scanning laser ophthalmoscopy (SLO). In all five carriers examined, qualitative and quantitative analyses by AO SLO imaging revealed a mosaic pattern of cone disruption, even in the absence of visual symptoms, normal visual acuity and normal macular thickness, on optical coherence tomography and mildly subnormal full-field cone electroretinographic findings. As the technique is sensitive to the level of a single cone, the ability to visualize the cone cells in vivo should be especially useful in other retinal diseases. In addition, further investigation of XLRP carriers may yield insight into how cone structures change over time and ultimately enable understanding of the role of $R P G R$ and $R P 2$ in cone cell survival.
\end{abstract}

European Journal of Human Genetics (2013) 21, 1240-1248; doi:10.1038/ejhg.2013.21; published online 27 February 2013

Keywords: X-linked retinitis pigmentosa carrier; photoreceptor layer; adaptive optics scanning laser ophthalmoscopy

\section{INTRODUCTION}

Identification of X-linked retinitis pigmentosa (XLRP) carriers is challenging and has serious implications for genetic counseling. Female XLRP carriers usually have normal, or close to normal, visual acuity, but often show some degree of fundus changes, including sectorial or peripheral pigmentary deposits and a tapetal-like reflex (TLR), ${ }^{1}$ as well as abnormal fundus. Many previous studies have detailed the prevalence of abnormal fundus photography, ${ }^{1,2}$ vitreous fluorophotometry, ${ }^{3}$ infrared (IR) reflectance images, ${ }^{1,2}$ electroretinographic (ERG), ${ }^{4,5}$ psychophysical $^{6}$ and flicker-fusion ${ }^{7}$ findings in such carriers. More recently, several studies have investigated XLRP carriers with advanced devices, including FAF, ${ }^{8}$ multifocal $\mathrm{ERG}^{9}$ and $\mathrm{OCT}^{10,11}$ to reveal clinical phenotypes of XLRP. Most XLRP carriers can be identified on the basis of fundus findings and ERG changes; however, $10-30 \%$ of carriers show no abnormal ERG or fundus finding. $1,4,5,9$

Although XLRP is the least common genetic type of RP, accounting for $6-17 \%$ of familial retinitis pigmentosa cases, it is extremely devastating. ${ }^{1,12,13}$ In the first or second decade of life, affected individuals experience a decrease in peripheral and night vision, and the disease often leads to partial or complete blindness in the fourth or fifth decade of life. ${ }^{14}$ In contrast to the severe retinal degeneration in affected men, female carriers of this genetic subtype show greater phenotypic variability, probably due to non-random or skewed inactivation of one $\mathrm{X}$ chromosome. ${ }^{15}$ Therefore, previous studies with novel and advanced equipment have been performed in female carriers of XLRP to offer insight into pathogenic mechanisms involved in this tragic affliction.
Previous genetic studies revealed two major genes have been identified - RPGR and RP2. ${ }^{16,17}$ However, the interpretation of genetic mutations and their correlation with the clinical phenotypes remains unknown, and the role of these genes in the photoreceptor cilia is not yet completely elucidated. ${ }^{18}$ A major reason for this is that it has not been possible to study individual photoreceptors affected by XLRP in living patients because irregularities of the human eye's optics limit the resolution of retinal images produced by all methods commonly used in clinical practice. ${ }^{19}$ Adaptive optics (AO) can compensate for these aberrations in living eyes by using a wavefront sensor to measure aberrations of the eye and a deformable mirror or a spatial light modulator to compensate for these aberrations. ${ }^{20,21}$ After the aberrations are corrected, lateral resolutions of the order of $2 \mu \mathrm{m}$ can be achieved, thereby allowing visualization of singlecone photoreceptors with the $\mathrm{AO}$ technology. ${ }^{22,23}$ The integration of AO with scanning laser ophthalmoscopy (SLO), that is, $\mathrm{AO}-\mathrm{SLO}$, provides images of the retina with a high resolution and contrast. $^{21,24}$

Recent studies with AO-SLO have revealed that this technique provides a unique opportunity to analyze the effects of genetic mutations on photoreceptors. ${ }^{19-25}$ To better understand the pathogenesis of XLRP, high-resolution images of the photoreceptor layer and AO-image-derived measures of photoreceptor integrity are essential. This study was designed to evaluate the structural characteristics in five female obligate carriers of XLRP by using various retinal imaging methods, including a compact AO-SLO that uses a dual liquid-crystal phase modulator introduced by Hirose et al. ${ }^{26}$ This non-invasive approach makes it possible to investigate the

${ }^{1}$ Department of Ophthalmology, Columbia University, New York, NY, USA; ${ }^{2}$ Department of Ophthalmology, Kangdong Sacred Heart Hospital, Hallym University Medical Center, Seoul, South Korea; ${ }^{3}$ Department of Pathology and Cell Biology, Columbia University, New York, NY, USA

*Correspondence: Dr SH Tsang, Department of Ophthalmology, Columbia University/Edward S. Harkness Eye Institute, 635 West 165 th Street, New York, NY 10032, USA Tel: +1 212342 1186; Fax: +1 212342 7942; E-mail: sht2@columbia.edu

Received 16 July 2012; revised 2 November 2012; accepted 24 January 2013; published online 27 February 2013 
structural abnormalities in individual photoreceptor cells in obligate XLRP carriers.

\section{MATERIALS AND METHODS}

A total of 5 patients and 18 controls having similar age and refractory error with patients were investigated; all subjects were informed about the aims of the examination before they volunteered to participate. XLRP was diagnosed by pedigree analysis, which showed an absence of male-to-male transmission, and an evaluation of affected men and clinical features of female family members. The study was approved by the Columbia University institutional review board (AAAII480 and AAAB6560). All individuals gave informed consent according to the tenets of the Declaration of Helsinki before participation in any of our studies.

A complete medical history was obtained from all patients and controls, including information about all known family members. All participants underwent a complete ophthalmic examination that included measurement of best-corrected visual acuity (BCVA), intraocular pressure, subjective refraction, indirect ophthalmoscopy, slit-lamp examination with a contact lens, color fundus photography, IR fundus imaging and fundus autofluorescence (FAF) imaging. En face IR and FAF images were obtained with the Spectralis HRA + OCT (Heidelberg Engineering, Heidelberg, Germany) together with high-resolution spectral domain (SD)-OCT images.

Pupils were dilated with topical $1 \%$ tropicamide before full-field ERG, which was recorded from both eyes with silver-impregnated fiber electrodes (DTL; Diagnosys LLC, Littleton, MA, USA) according to the International Society for Clinical Electrophysiology of Vision (ISCEV) standards for both scotopic and photopic states. ${ }^{27}$

The patients who consented to give blood samples were genetically screened. Total genomic DNA was extracted from peripheral leukocytes with the QIAamp DNA Mini Kit (Qiagen, Valencia, CA, USA). The extracted genomic DNA was amplified by polymerase chain reaction (PCR) with previously delineated primer sequences at annealing temperatures. ${ }^{28}$ The PCR products were purified (QIAquick PCR Purification Kit; Qiagen) and sequenced.

All carriers and 18 age-matched participants with healthy eyes underwent AO-SLO. As has been previously described by Hirose et $a l,{ }^{26}$ components of the AO-SLO system include the AO system, light sources for imaging and measuring aberrations, an optical system, a scanner, and the data acquisition and image composition systems. Two light sources were used for this equipment. One was a superluminescent diode (S840-B-I-20; Superlum, Carrigtwohill, Ireland) with a wavelength of $840 \pm 25 \mathrm{~nm}$ for imaging. The other (a beacon light) was a superluminescent diode (QSDM-760-2; QPhotonics, Ann Arbor, MI, USA) with a wavelength of $760 \pm 5 \mathrm{~nm}$ for measuring aberrations. By calculating the incident power of light sources according to the American National Standards Institute safe light exposure levels, the power of the imaging light was set at $450 \mu \mathrm{W}$ and that of the beacon light, at $40 \mu \mathrm{W}$.

The AO subsystem contains a dual liquid crystal on the silicon spatial light modulators (LCOS-SLMs; X10468-02; Hamamatsu Photonics, Hamamatsu City, Japan) with an active area of $16 \times 12 \mathrm{~mm}$ and $792 \times 600$ pixels and a maximum stroke of $1 \lambda(840 \mathrm{~nm})$. A customized software controls the dual liquid crystal spatial light modulator and the wavefront sensor to compensate for higher-order aberrations.

The polarized components of the imaging light to the wavefront aberrations, caused by optical irregularities in the eye, were modulated by two LCOS-SLMs. The beacon light and a Hartmann-Shack wavefront sensor (HASO 32-eye; Imagine Eyes, Orsay, France) was used to measure these aberrations. Horizontal raster scans were created by an $8-\mathrm{kHz}$ resonant scanner (CRS $8 \mathrm{kHz}$; GSI Group, Bedford, MA, USA) and a galvanometric beam scanner (VM500 + ; GSI Group) was used to scan the imaging beam in the horizontal direction. When the imaging light was scanned, the data acquisition system of the reflected light system was initialized. An avalanche photodiode detected the light reflected from the retina and the light converted to an electrical signal. The electrical signal was then amplified 10 -fold by a commercial amplifier (SR560; Stanford Research Systems, Sunnyvale, CA, USA). Simultaneously, the signal from the amplifier was digitized by an Analog/Digital (AD) converter board. A personal computer recorded the digitized data to its hard disk over a period of approximately $2 \mathrm{~s}$. The final SLO image size was $400 \times 400$ pixels (32 frames/s).

The eye with better visual acuity or more stable fixation was chosen for imaging studies. We obtained the images at multiple locations within $1.5 \mathrm{~mm}$ of the center of fovea and created a montage of AO-SLO images by selecting the area of interest and generating each desired image. Each montage of the area of interest was verified by comparing the AO-SLO image with wide-field images and examining its correspondence. The cone density was measured in areas that were $0.5,1.0$ and $1.5 \mathrm{~mm}$ from the center of the fovea in each of the four directions (superior, inferior, nasal and temporal). We selected these distances because the our $\mathrm{AO}$ system cannot clearly show individual cones at the center of the fovea; this limitation has also been reported with other similar systems. ${ }^{20-24,26,29,30}$ Cone cells at foveal eccentricities of $>0.15 \mathrm{~mm}$ can be resolved.

To measure cone density, the number of cones was counted in the area of AO-SLO images. The cone cells appeared as bright spots that were $2-5 \mu \mathrm{m}$ in diameter, and the examiners manually selected areas $(50 \times 50$ pixels $)$, while excluding the areas where in retinal vessels or artifacts obscured the underlying cone mosaic. That is, none was obscured by blood vessels and artifact. We manually counted the number of cones within that area by placing the computer cursor on the image and clicking on it, to ensure that no cone was counted twice and that none was excluded within a selected area in the registered images. And after that, we used the photoreceptor analysis software (developed by Canon, Inc., Tokyo, Japan) for automated cone selection and measurement of the cone density within the selected area in the registered images. The selected area was same as manual method in which cone cells were not obscured by blood vessels and artifact. Cone density, the number of cones divided by the area of retina sampled, was estimated automatically by that software. As a result, we acquired two kinds of parameters for the number of cones: cone count by manual estimation and cone density by automated estimation.

\section{RESULTS}

The groups of patients $(n=5)$ and normal volunteers $(n=18)$ who participated in this study were all females and had BCVA of 20/20 or better in at least one eye. The mean age of the carriers was $39.8 \pm 8.4$ and control was $44.8 \pm 9.88$. As the packing density of cones does change as a function of age and refractory error, ${ }^{29,30}$ subject 4 was separated from the other carriers to compare with controls whose age range from 50 to 58 and refractory error range from $-7.0 \mathrm{D}$ to $-8.5 \mathrm{D}$. Table 1 summarized carrier's profiles and the details of their examined eyes including vision and there was no underlying disease except carrier of XLRP in our patients.

All of patients were screened for mutations in the RP2 and $R P G R$ genes, and all of the exons were sequenced after the genomic DNA was amplified. The results are indicated in Table 2. Although four of our patients confirmed having mutations in RPGR gene, we did not get a genetic result from one further patient. However, subject 3 had one son with advanced RP, two normal daughters, two normal sons and a normal husband. Subject 3's two brothers had advanced RP but there was no clinical evidence of RP in her parents. None of her sisters or their children had RP. Subjects 1 and 5 were sisters from the same parents.

On slit lamp examination, the cornea and anterior chamber were clear and no patients showed lens opacity or inflammation. Of our five carriers, a TLR was seen in all patients. The reflex was associated with fundus changes seen in high myopia such as prominence of the choroidal vascular pattern (Figure 1a). Within the area of the TLR, FAF image showed speckled regions of enhanced AF (Figure 1b) and IR image displayed an enhanced reflectivity (Figure 1c). None of the patients had peripheral pigment atrophy or bone spicule-like pigmentary clumping. Full-field ERG response from eyes tested on the AO-SLO is summarized in Table 3 . For $30 \mathrm{~Hz}$ flicker stimulus ERG, three of five carriers (subjects 2, 3 and 4) showed mild reduction in 
Table 1 Patient and ocular characteristics

\begin{tabular}{|c|c|c|c|c|c|}
\hline & Subject 1 & Subject 2 & Subject 3 & Subject 4 & Subject 5 \\
\hline Sex & Female & Female & Female & Female & Female \\
\hline Age (years) & 34 & 39 & 33 & 54 & 29 \\
\hline Ethnicity & Caucasian & Asian & Caucasian & Caucasian & Caucasian \\
\hline Imaged eye & OS & OD & OS & OD & OS \\
\hline BCVA & $20 / 20$ & $20 / 20$ & $20 / 20$ & $20 / 20$ & $20 / 20$ \\
\hline \multirow[t]{2}{*}{ Subjective refraction } & Sph: -4.5 & Sph: -1.0 & Sph: -1.25 & Sph: -7.75 & Sph: -0.25 \\
\hline & Cyl: -3.75 & Cyl: -0.25 & Cyl: -0.25 & Cyl: -1.0 & Cyl: -1.75 \\
\hline Axial length (mm) & 25.89 & 25.75 & 22.52 & 24.82 & 24.22 \\
\hline ACD depth (mm) & 3.55 & 3.5 & 2.88 & 3.68 & 3.43 \\
\hline
\end{tabular}

Abbreviations: ACD, anterior chamber depth; BCVA, best-corrected visual acuity.

Table 2 Genetic results of patients

\begin{tabular}{llllll}
\hline & Subject 1 & Subject 2 & Subject 3 & Subject 4 & Subject 5 \\
\hline Gene & RPGR & RPGR & Pending & RPGR & RPGR \\
Exon & ORF 15 & ORF 2 & ORF 11 & ORF 15 \\
Condon change & Glu938X & Gly68Arg & Gly436Asp & Glu938X \\
Nucleotide change & $2812 \mathrm{G}>\mathrm{T}$ & $202 \mathrm{G}>\mathrm{A}$ & & $1307 \mathrm{G}>\mathrm{A}$ & $2812 \mathrm{G}>\mathrm{T}$ \\
\hline
\end{tabular}

b-wave amplitude and two carriers (subjects 1 and 4) found having an implicit time delay. For single-cone response, only one carrier (subject 5) showed slightly reduced b-wave amplitude and also only one carrier (subject 3) mild reduction in b-wave amplitude for rod-specific ERG. For maximal dark-adapted rod-cone response, only one carrier (subject 2) showed a mildly prolonged implicit time, whereas none of the carriers showed an abnormal finding in b-wave amplitude. Using SD-OCT, all carriers were revealed that had normal macular structure, with an intact inner segment (IS)/outer segment (OS) junction and grossly normal appearing outer nuclear layer plus outer plexiform layer $(\mathrm{ONL}+)$ thickness within the posterior pole (Figure 2).

Regarding AO-SLO examination, the cone photoreceptors of normal subjects showed the appearance of slightly oval-shaped bright dots with a compact arrangement (Figures $3 e$ and f, Figures $4 \mathrm{e}-\mathrm{h}$ ). Manual (cone count) and automatic (cone density) estimation of number of normal cone cells decreased with increasing eccentricity from the center of fovea (Tables 4 and 5). High-resolution macular cone images of all five carriers using AO-SLO were highly variable, both in image quality and in the appearance of mosaic itself. Compared with normal eyes, some images of each patient showed normal appearance of cone cell with a compact arrangement (Figure 3d). However, cone cells of several areas showed irregular pattern of cone cells and were of variable asymmetrical sizes and shapes. There were some dark areas where the cone cells were damaged or lost (Figures $3 \mathrm{f}$ and $4 \mathrm{a}-\mathrm{d}$ ), conducing less compact distribution of cone cells than controls. These patterns were not observed in unaffected retina (Figures $4 \mathrm{e}-\mathrm{h}$ ) in same eccentricity and differentiated with dark areas of artifact and blood vessel. When we manually counted the number of cone cells in these images, cone count was reduced, compared with unaffected controls.

To demonstrate the patter of cone cell distributions in our patients, the number of cone cells was calculated manually in each retinal image and is summarized in Table 4. Comparison of the cone count between the four XLRP carriers (subjects 1, 2, 3 and 5) and healthy eyes (age from 30 to 48 , refractory error from $-0.25 \mathrm{D}$ to $-4.75 \mathrm{D}$, $n=12$ ) showed significant decreases at a distance of $0.5 \mathrm{~mm}$ from the center of the fovea in all directions and $1.0 \mathrm{~mm}$ from the fovea in three directions (inferior, temporal and nasal). There was no significant difference between normal controls and four carriers at a distance of $1.5 \mathrm{~mm}$ from the fovea in all directions except temporal $1.5 \mathrm{~mm}$ to the fovea. In comparison between subject 4 and controls (age from 50 to 58 , refractory error from $-7.0 \mathrm{D}$ to $-8.5 \mathrm{D}, n=6$ ), none of statistical difference was present in all eccentricities. However, when comparing cone count in all of the each area with average value of controls, inferior $0.5 \mathrm{~mm}$ to the foveal center in subject 4 showed lower cone count than 1 SD from average of age-matched controls. In addition to quantitative differences, qualitative abnormalities with irregular cone cells were observed in AOSLO image (Figures $4 \mathrm{a}-\mathrm{d}$ ).

A result of cone density for all carriers examined is shown in Table 5. The number of cone cells was counted using photoreceptor analysis software that automatically counted the cones within the selected area in the registered images. Compared with the normal eyes, ranging from 30 to $48(n=12)$, there were significant decreases at a distance of $0.5 \mathrm{~mm}$ in all directions and $1.0 \mathrm{~mm}$ in 2 directions (nasal and temporal) from the fovea. As same as cone count, there was no significant difference between normal controls and four carriers at a distance of $1.5 \mathrm{~mm}$ from the fovea in all directions except temporal $1.5 \mathrm{~mm}$ to the fovea. In comparison between subject 4 and controls $(n=6)$, there was no significant difference in all eccentricities. Different from the result of manual estimation, nasal $0.5 \mathrm{~mm}$ to the fovea in subject 1 , temporal $1.5 \mathrm{~mm}$ to the fovea in subject 2 and superior $1 \mathrm{~mm}$ to the fovea and nasal $0.5 \mathrm{~mm}$ to the fovea in subject 5 showed lower cone density than 1 SD from the average value of the normal. Entirely, 29 areas showed cone counts or densities $<1$ standard from the controls in either manual or autonomic method and 24 areas (82.8\%) were overlapped.

\section{DISCUSSION}

XLRP carriers have a broad spectrum of fundus changes, ranging from normal fundus to extensive retinal degeneration; they commonly show a TLR or peripheral pigmentary disturbance. ${ }^{31} \mathrm{~A}$ previous study revealed that presence of TLR is independent of mutation, ${ }^{8}$ but it fades with age and tends to be most apparent in younger women aged below 40 years. ${ }^{1,31}$ Enhanced reflectivity or AF was found within the area of TLR and OCT showed normal retinal microstructure and thickness, but an increased reflectivity was observed from the RPE-photoreceptor layer complex. ${ }^{1,8,10}$ Peripheral pigmentary retinal degeneration was not observed in any of our carriers, but myopic fundus changes such as prominence of the choroidal vascular pattern was present in three carriers. 


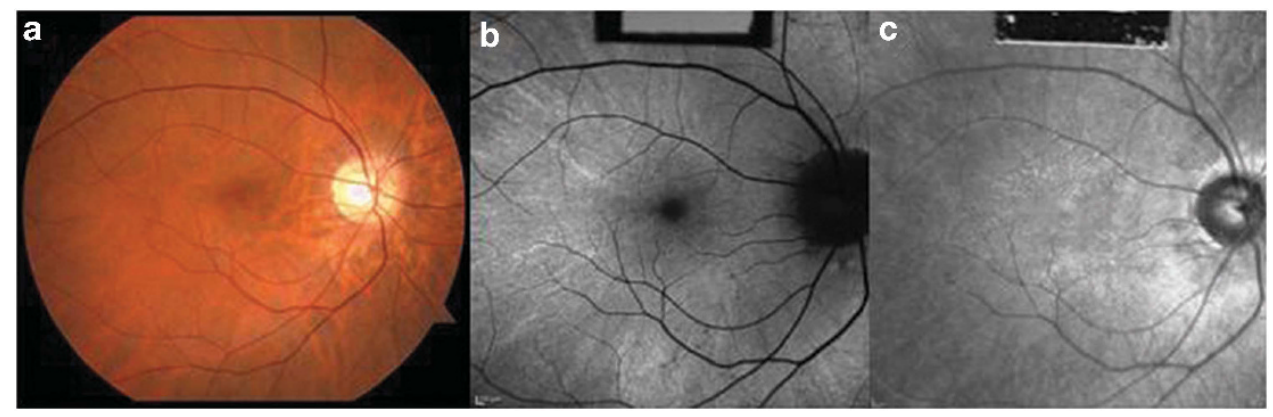

Figure 1 Retinal images of the left eyes of XLRP carrier. (a) Fundus photograph of subject 2 shows fundus TLR within the posterior pole with tigroid changes. (b) Hyperautofluorescences are detected within the area of the TLR. (c) IR image showing an enhanced reflectivity that correlated with the fundus TLR. AF, autofluorescence.

Table 3 The result of XLRP carriers for the standard full-field electroretinogram

\begin{tabular}{|c|c|c|c|c|c|c|c|c|}
\hline Carrier & $\begin{array}{c}\text { Cone } \\
\text { response } \\
\text { (amplitude) }\end{array}$ & $\begin{array}{c}\text { Cone } \\
\text { response } \\
\text { (implicit time) }\end{array}$ & $\begin{array}{c}\text { Rod } \\
\text { response } \\
\text { (amplitude) }\end{array}$ & $\begin{array}{c}\text { Rod } \\
\text { response } \\
\text { (implicit time) }\end{array}$ & $\begin{array}{l}\text { Maximum } \\
\text { response } \\
\text { (amplitude) }\end{array}$ & $\begin{array}{c}\text { Maximum } \\
\text { response } \\
\text { (implicit time) }\end{array}$ & $\begin{array}{c}30 \mathrm{~Hz} \\
\text { flicker } \\
\text { (amplitude) }\end{array}$ & $\begin{array}{c}30 \mathrm{Hs} \\
\text { flicker } \\
\text { (implicit time) }\end{array}$ \\
\hline Subject 1 & 90.1 & 23.2 & 133.4 & 89.2 & 194.2 & 46.5 & 77.35 & $27.4^{a}$ \\
\hline Subject 2 & 84.73 & 25.3 & 144.6 & 95.1 & 190.2 & $50.6^{a}$ & $62.3^{b}$ & 26 \\
\hline Subject 3 & 85.4 & 28.4 & $103^{b}$ & 91.6 & 208.1 & 47.3 & $65.8^{b}$ & 26.1 \\
\hline Subject 4 & 82.62 & 29.2 & 184.3 & 76.2 & 277.8 & 46 & $63.33^{b}$ & $28^{a}$ \\
\hline Subject 5 & $81.2^{\mathrm{b}}$ & 29 & 125.2 & 77.6 & 265.4 & 48.2 & 76.23 & 24.2 \\
\hline Normal $^{\mathrm{c}}$ & $128.7 \pm 46.9$ & $27.75 \pm 1.73$ & $241.1 \pm 116.6$ & $89.67 \pm 16.5$ & $333.2 \pm 201.1$ & $41.7 \pm 8.7$ & $108 \pm 32.66$ & $24.33 \pm 1.97$ \\
\hline
\end{tabular}

The full-field electroretinogram (b-wave) results were reported in eyes tested on the adaptive optics scanning laser ophthalmoscopy.

almplicit time delayed than 1 SD from average implicit time of normal.

${ }^{\mathrm{b}}$ Amplitude $<1$ SD from average amplitude of normal.

Normal range for full-field electroretinogram system.

The pathogenic mechanisms of XLRP remain uncertain because only a few histological studies on young XLRP donors have been previously conducted. In 1985, autopsy eyes were first examined from a 79-year-old female carrier who had no visual symptoms and had normal visual acuity. ${ }^{32}$ In this study of young individuals, the rods and cones in the posterior pole were within the normal limit, and scattered circular or oval patches of photoreceptor cell degeneration were observed in a midperipheral annulus. However, a recent study of an XLRP carrier with an RPGR mutation showed patches with abnormal or no photoreceptors and diminished numbers of cone cells in parafoveal and perifoveal regions, although she had a normal visual acuity. ${ }^{33}$ Another histological study of a carrier who had a normal fundus showed similar results with loss of some photoreceptor cell nuclei observed in the carrier but not in controls. ${ }^{34}$

AO-SLO provides a means of performing an 'optical biopsy' and to study live patients at the cellular level. Our novel findings with AOSLO were more comparable to the results from histological analyses of two recent histological studies than those of an earlier one. ${ }^{32-34}$ Despite normal visual acuity and mildly subnormal ERG findings, every carrier had several subnormal areas, where distribution of cones were less compact, and abnormal cone cells, which appeared to form mosaic patterns from their size and shape. What is intriguing is the fact that there were no specific SD-OCT findings on all carriers, highlighting that it may not be possible to directly deduce the degree of cone cell structure from SD-OCT imaging alone.

In both manual and automatic cone counting, statistical analysis between four carriers of a similar age and controls revealed that the number of cone cells was significantly decreased at a distance of
$0.5 \mathrm{~mm}$ but not $1.5 \mathrm{~mm}$ from the fovea. Measurements $1.0 \mathrm{~mm}$ inferior to the fovea only indicated significant differences between controls and carriers by using the manual method, but not by the automated method. When we compared the average number of cone cells counted using either method, 29 areas showed cone counts or densities $<1$ SD from the controls and, among these areas, 16 areas were $0.5 \mathrm{~mm}$ from the fovea regardless of direction. Qualitatively, the areas with subnormal cone counts or densities appeared to have more dark areas where cone cells were not existed or severe cone cell irregularities than those with normal cone counts or densities and the agreement between the two different methods was up to $82.8 \%$. These results indirectly indicate that automatic measurement of cone density is as useful as manual method for cone cell calculating. However, we could not clearly confirm these observations because of the variability in the quality of images and small sample size. Therefore, further evaluation including reproducibility of automated method must be needed.

Even we only obtained 12 areas of AO-SLO images in individual patient, both subnormal and normal areas were found to spontaneously exist in the same patient with dysmorphic cone cells, which also distributed irregularly within the whole areas. These results can be supported by random X-inactivation hypothesis suggested by Lyon. ${ }^{35}$ In early embryonic development, one X-chromosome in each cell of a woman becomes inactive. This inactivation occurs in a random manner and is independent in each cell. Uneven lyonization has been known as a possible cause for a discordant phenotypical expression of X-linked diseases. ${ }^{36}$ This hypothesis implies that the affected cone cell has a skewed inactivation predominantly of the 


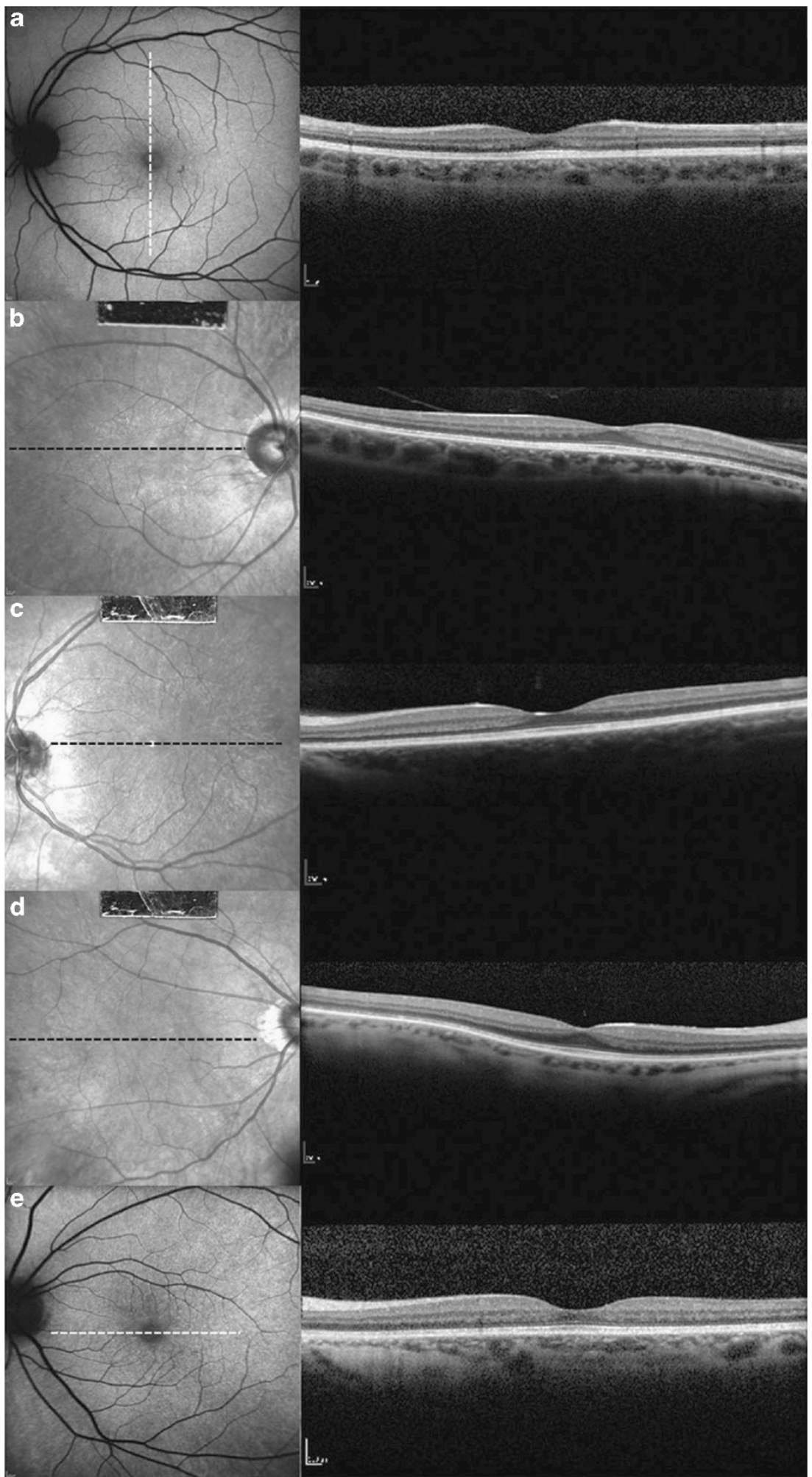

Figure 2 High-resolution SD-OCT scans through the fovea of the five XLRP carriers. SD-OCT images of (a) subject 1, (b) subject 2, (c) subject 3, (d) subject 4 and (e) subject 5. All carriers with TLR have normal retinal microstructure and thickness on SD-OCT images. These SD-OCT images also showed an intact inner ellipsoid band, indicating the presence of photoreceptor IS/OS junctions. Photoreceptor OSs, RPE and Bruch's membrane were also intact in all carriers. The position of the SD-OCT line scan is indicated by the white or black dashed lines in AF or IR images. AF, autofluorescence; RPE, retinal pigment epithelium; SD-OCT, spectral domain optical coherence tomography. 


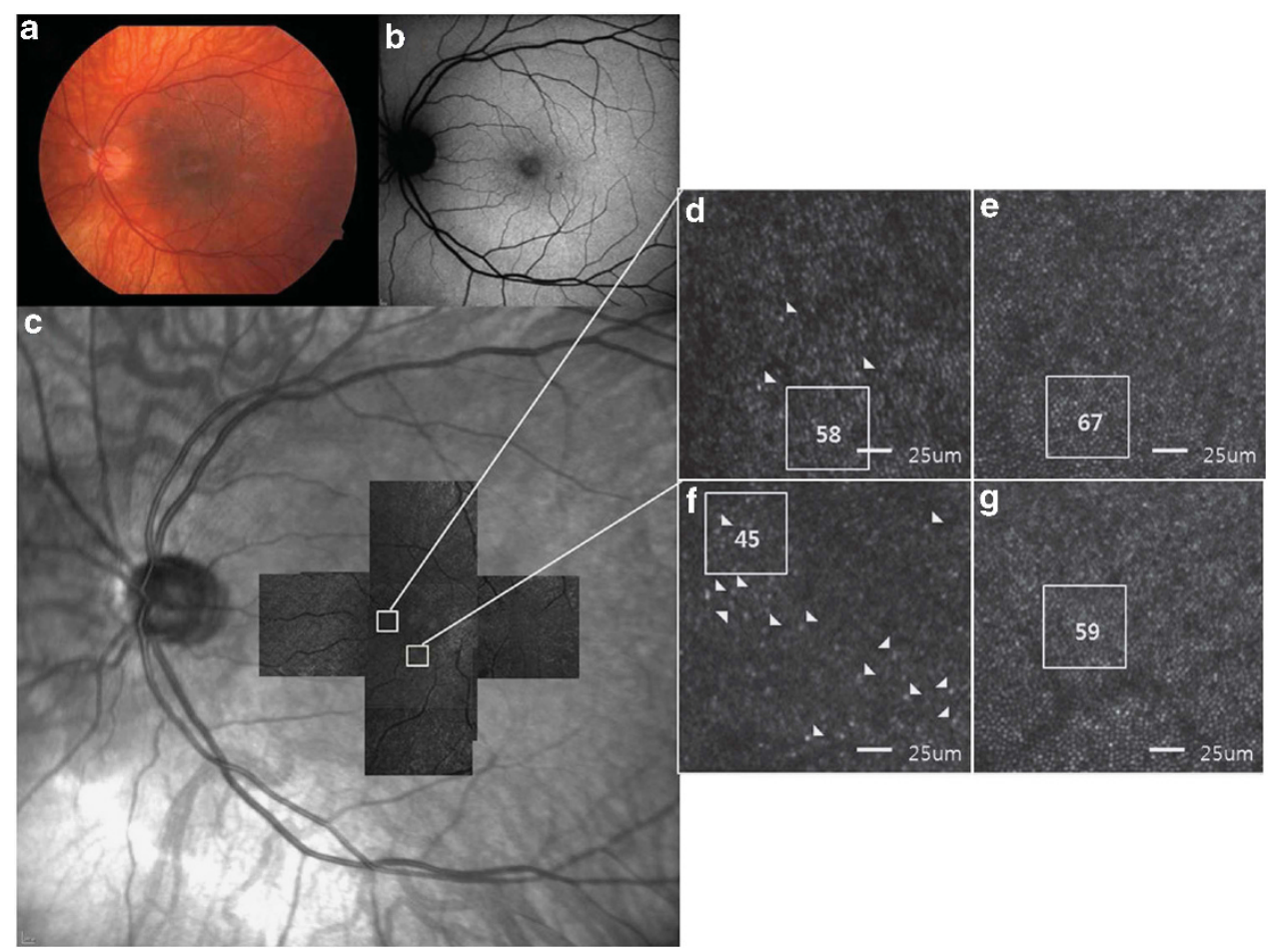

Figure 3 Retinal images of the left eye of subject 1. (a) Fundus photograph shows fundus TLR within the posterior pole, which is more prominent temporal to the macula, and myopic changes with prominence of the choroidal vascular pattern. (b) No abnormal AF is detected on FAF imaging. (c) A montage of AO-SLO images from subject 1 matched with the IR images. (d) A magnified AO-SLO image of the area (nasal $0.5 \mathrm{~mm}$ ) indicated by the white box in the image of c, showing a relatively regular cone cell appearance. Some morphologically large and/or dysmorphic cone cells are indicated by arrowheads, but it is not that much compared with another area (inferior $0.5 \mathrm{~mm}$ ) in the same patient. The number of cone cells within the area indicated by the white box was estimated to be 58. (e) A magnified AO-SLO image from an unaffected control at the same location. (f) A magnified AO-SLO image of the area (inferior $0.5 \mathrm{~mm}$ ) indicated by the white box in the image of $\mathbf{c}$, shows less compact distributions than those with normal controls. Some cone cells show irregular appearance and are of variable asymmetrical sizes and shapes, which are indicated by arrowheads and the cone count is 45 in the white box area. (g) A magnified AO-SLO image from an unaffected control at the same location.

wild-type X-chromosome, whereas the unaffected cone cell either has a random $\mathrm{X}$-inactivation or a predominant inactivation of the $\mathrm{X}$-chromosome carrying the mutant gene.

The RPGR gene, which account for about $70 \%$ of all XLRP cases, is located in chromosomal region Xp21.1, spans $172 \mathrm{~kb}$ and was initially reported to contain 19 exons. ${ }^{18}$ In retina, the gene product is located in the connecting cilium of rods and cones, involved in cellular trafficking and has a role in transGolgi transport. ${ }^{8}$ Compromised intracellular transport of major components of the phototransduction cascade including rhodopsin results from loss of $R P G R$ function, thus impairing proper formation of OSs and leading to subsequent loss of photoreceptors by apoptosis. Mutations can be found in the first 14 exons in $20-30 \%$ of all XLRP patients and in a further $50-80 \%$ of patients in the ORF15 exon, thus ORF15 is known as a mutational hotspot. ${ }^{18}$ Despite a broad overlap in phenotype between RP patients with RPGR mutations, previous study showed that patients with mutation in exons 1-14 had a smaller visual filed area, lower ERG amplitude and lost ERG amplitude 50\% greater than those with ORF15 mutations. ${ }^{37,38}$ In contrast to these studies, subject 4 with ORF11 mutation had milder AO-SLO features, in terms of both quantity and quality, along with better ERG amplitude than subjects 1 and 5 who had ORF15 mutations, even subject 4 was older and high myopia. Although this result showed that the AO-SLO finding well matched with ERG, proving sensitivity of AO-SLO.

Some limitations of this study should be noted. RP is a disease predominantly involving the rod photoreceptor system.1,12-14
However, the current AO-SLO system used in our study could not assess the rod photoreceptor system qualitatively or quantitatively. Another limitation is that RP starts in the mid-periphery and advances toward the macula and fovea, ${ }^{1,12-14}$ but we were unable to obtain images of the peripheral retina and fovea using the current AO-SLO system. Although random X-inactivation hypothesis can explain a mosaic distribution of abnormal cone cells, these limitations make us unable to exactly explain the reason for subnormal areas being predominantly observed at a distance of $0.5 \mathrm{~mm}$ from the fovea.

Nevertheless, recent studies revealed that RPGR defect is a common cause of cone-rod dystrophy and observed that mutations in $R P G R$ can cause cone-specific degeneration, indicating that in addition to a well-established role for rod cells, RPGR has an important role in cone photoreceptors. ${ }^{39-41}$ Studies with knockout mice lacking the RPGR gene exhibit cone opsin mislocalization that is evident from early ages onward. ${ }^{42}$ For mice in which Rpqr exon 4 was deleted, extent of rod and cone involvement depended on the genetic background with cone disease predomination on the albino BALB/cJ background. ${ }^{43}$ From these background, we can draw a conclusion that the distance of $0.5 \mathrm{~mm}$ from the fovea have the highest density of cones with lowest density of rods, so that cone cells were easily and primarily affected by RPGR defect. Random X-inactivation may have a role in this phenomenon and that can be also associated with TLR, which generally exist within the posterior pole rather than peripheral area. ${ }^{10}$ However, the reason for this phenomenon in cone cell survival and abnormality remains elusive and it will be important 

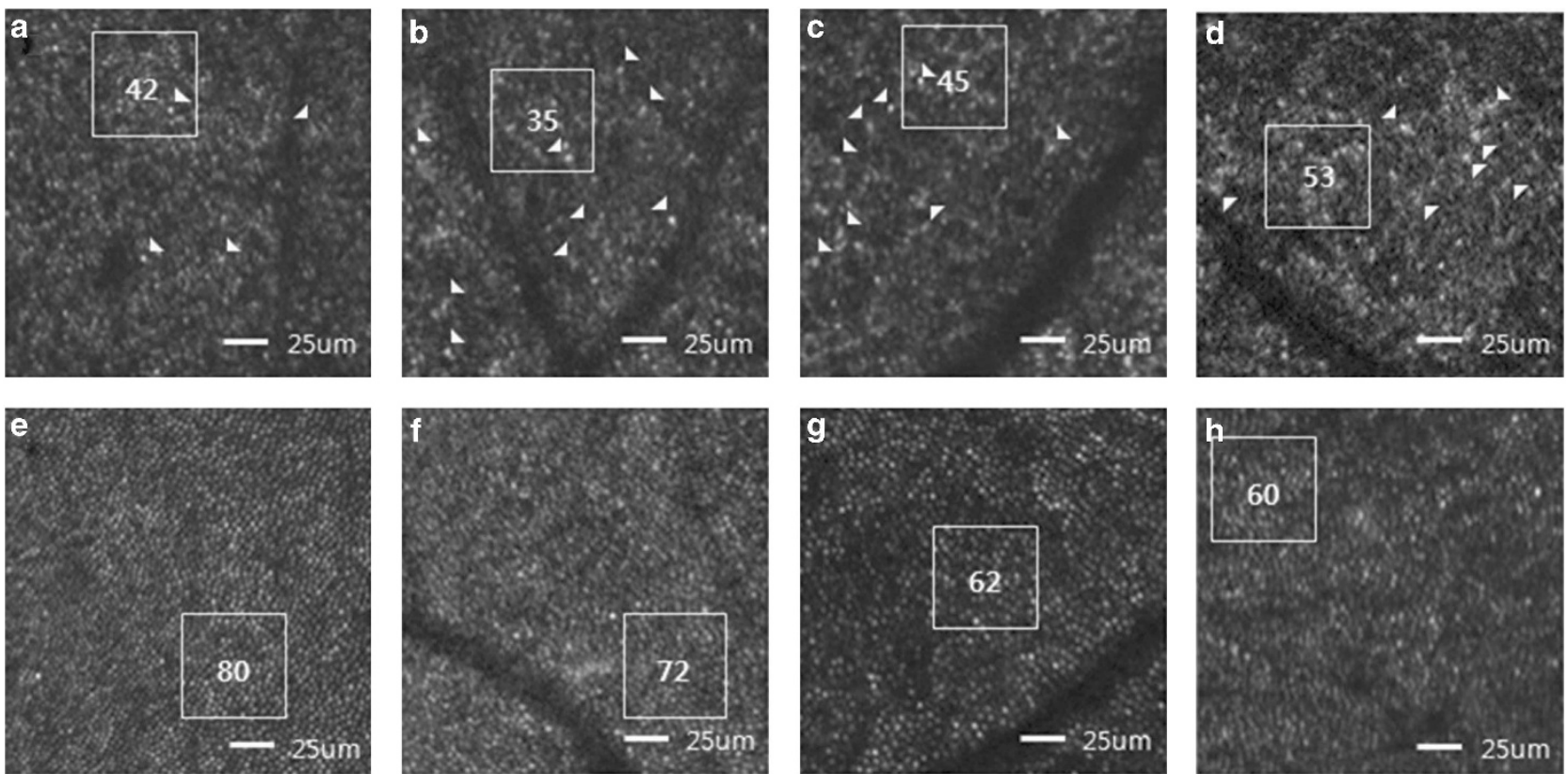

Figure 4 AO-SLO images of carriers of XLRP and controls. Manual counting of the number of cone cells was performed within the area indicated by white box. Some morphologically large and/or abnormal cone cells are indicated by arrowheads. (a) AO-SLO image of subject 2 (temporal 0.5 mm). (b) AO-SLO image of subject 3 (temporal $1.0 \mathrm{~mm}$ ). (c) AO-SLO image of subject 5 (inferior $0.5 \mathrm{~mm}$ ). (d) AO-SLO image of subject 4 (inferior $0.5 \mathrm{~mm}$ ). Presented images of all carriers have several small patch lesions where cones are lost or decreased and residual cone cells appear to be asymmetrical sizes and be of variable shapes. (e, f, $\mathbf{g}, \mathbf{h}$ ) AO-SLO images from an unaffected control at the same location and magnification used for XLRP carriers. The images show normal cone mosaic patterns and no abnormal patch lesions.

Table 4 Cone count of the patients with X-linked retinitis pigmentosa carrier

\begin{tabular}{|c|c|c|c|c|c|c|c|c|c|c|c|c|}
\hline Carrier & $\begin{array}{l}\text { Superior } \\
0.5 \mathrm{~mm}\end{array}$ & $\begin{array}{l}\text { Superior } \\
1.0 \mathrm{~mm}\end{array}$ & $\begin{array}{c}\text { Superior } \\
1.5 \mathrm{~mm}\end{array}$ & $\begin{array}{l}\text { Inferior } \\
0.5 \mathrm{~mm}\end{array}$ & $\begin{array}{l}\text { Inferior } \\
1.0 \mathrm{~mm}\end{array}$ & $\begin{array}{l}\text { Inferior } \\
1.5 \mathrm{~mm}\end{array}$ & $\begin{array}{c}\text { Temporal } \\
0.5 \mathrm{~mm}\end{array}$ & $\begin{array}{c}\text { Temporal } \\
1.0 \mathrm{~mm}\end{array}$ & $\begin{array}{c}\text { Temporal } \\
1.5 \mathrm{~mm}\end{array}$ & $\begin{array}{l}\text { Nasal } \\
0.5 \mathrm{~mm}\end{array}$ & $\begin{array}{l}\text { Nasal } \\
1.0 \mathrm{~mm}\end{array}$ & $\begin{array}{l}\text { Nasal } \\
1.5 \mathrm{~mm}\end{array}$ \\
\hline $\begin{array}{l}\text { Subject } \\
1\end{array}$ & $51^{a}$ & 37 & 30 & $49^{a}$ & $30^{a}$ & 30 & $45^{a}$ & $33^{a}$ & 29 & 64 & 42 & 47 \\
\hline $\begin{array}{l}\text { Subject } \\
2\end{array}$ & $32^{a}$ & $30^{a}$ & 32 & $41^{a}$ & $35^{a}$ & 32 & $42^{a}$ & $32^{a}$ & 29 & $41^{a}$ & $38^{a}$ & 38 \\
\hline $\begin{array}{l}\text { Subject } \\
3\end{array}$ & $54^{a}$ & 39 & 32 & $48^{a}$ & $32^{a}$ & 38 & $55^{a}$ & $35^{a}$ & 35 & $51^{a}$ & 40 & 37 \\
\hline $\begin{array}{l}\text { Subject } \\
5\end{array}$ & $48^{a}$ & 44 & 45 & $48^{a}$ & 42 & 42 & $45^{a}$ & $40^{a}$ & 29 & 57 & 43 & 42 \\
\hline$P$-value ${ }^{\mathrm{b}}$ & 0.002 & 0.635 & 0.839 & 0.002 & 0.024 & 0.733 & 0.002 & 0.002 & 0.024 & 0.014 & 0.024 & 0.635 \\
\hline $\begin{array}{l}\text { Subject } \\
4\end{array}$ & 57 & 49 & 45 & $53^{a}$ & 44 & 33 & 54 & 53 & 44 & 62 & 38 & 41 \\
\hline$P$-value ${ }^{c}$ & 0.643 & 0.429 & 0.286 & 0.041 & 1 & 0.857 & 0.286 & 0.143 & 0.286 & 1 & 0.643 & 0.429 \\
\hline Average & $48.4 \pm 9.8$ & $39.8 \pm 7.2$ & $36.8 \pm 7.5$ & $47.8 \pm 4.3$ & $36.6 \pm 6.1$ & $35 \pm 4.9$ & $48.2 \pm 5.9$ & $38.6 \pm 8.6$ & $33.2 \pm 6.6$ & $55 \pm 9.3$ & $40.2 \pm 2.3$ & $41 \pm 3.9$ \\
\hline Normal $^{\mathrm{b}}$ & $67.5 \pm 5.5$ & $39.9 \pm 3.9$ & $33.2 \pm 3.3$ & $63.7 \pm 5.8$ & $43.4 \pm 3.3$ & $33.9 \pm 3.9$ & $68.5 \pm 10.7$ & $47.1 \pm 5,45$ & $40.8 \pm 11.3$ & $69.2 \pm 11.2$ & $47.6 \pm 7.9$ & $39.8 \pm 8.2$ \\
\hline Normalc & $52.3 \pm 8$ & $33.5 \pm 3.4$ & $33.2 \pm 3.6$ & $54.5 \pm 1.1$ & $38.8 \pm 3.9$ & $33.9 \pm 6$ & $54.7 \pm 9.1$ & $41.5 \pm 7.2$ & $34.5 \pm 5.7$ & $53.7 \pm 3.5$ & $39.2 \pm 4.9$ & $33.7 \pm 1.3$ \\
\hline
\end{tabular}

aCone count $<1$ SD from average cone count of age-matched control.

bStatistical analysis with age-matched 12 normal controls (mean age: $38 \pm 5.92$, refractory error: $-2.35 \pm 1.69$, Mann-Whitney U-test).

${ }^{c}$ Statistical analysis with age-matched six normal controls (mean age: $55 \pm 3.71$, refractory error: $-7.68 \pm 0.6$, Mann-Whitney $U$-test).

to develop a more complete understanding of the role(s) of $R P G R$ gene by comparing genotype-phenotype variations with AO-SLO system.

Although the number and structure of cones were disrupted, our carriers showed normal visual acuity and had tenuous abnormal ERG findings. Multiple but small foci of retinal disruption may not alter the full-field ERG and visual acuity, as these reflect the massed responses from the entire retina. ${ }^{8}$ However, previous studies with multifocal (mf) ERG presented a mosaic pattern of mfERG dysfunction in an XLRP carrier, although no clinically apparent retinal changes, retinal sensitivity losses on visual field testing, or abnormal full-field ERG response were observed. ${ }^{9}$ Thus, future studies on XLRP carriers should combine AO-SLO images with not only mfERG but also micoperimetry or visual-field tests to facilitate functional and structural assessments.

$\mathrm{XLRP}$ is the least common but most devastating form of RP. ${ }^{1,12-14}$ $R P G R$ and $R P 2$ have been implicated in XLRP. ${ }^{16,17}$ The diagnosis of XLRP has recently improved because of the introduction of DNA 
screening tests for these two genes. However, we have little information about genotype-phenotype variation of these two genes and their role in ciliary transport, especially in the carriers of presymptomatic XLRP. Therefore, we investigated five obligate carriers of XLRP by using divergent retinal imaging methods, including SD-OCT and novel AO-SLO system. Our study is significant because it is the first in vivo study to demonstrate the cone structure and estimate the number of cone cells in XLRP carriers. In all of our five carriers, a mosaic pattern of cone disruption was present even with normal visual acuity, macular thickness on SD-OCT, and full-field cone ERG findings, and in the absence of visual symptoms. We demonstrated that thorough analysis of AOSLO images could be a sensitive method to detect mosaicism in XLRP carriers in the early stages of the disease, and provide in vivo genotype-phenotype correlations in patients with known genetic mutations.

\section{CONFLICT OF INTEREST}

The authors declare no conflict of interest.

\section{ACKNOWLEDGEMENTS}

Publication of this article was supported in part by the P30EY019007 (Core Support for Vision Research; SHT) Grants EY018213 (SHT) and EY019861 (SHT) from the National Eye Institute, National Institutes of Health, Bethesda, MD, USA; Foundation Fighting Blindness, Owings Mills, MD, USA; and unrestricted funds from Research to Prevent Blindness Inc., New York, NY, USA. SHT is a Fellow of the Burroughs-Wellcome Program in Biomedical Sciences, and has been supported by the Bernard Becker Association of University Professors in Ophthalmology Research to Prevent Blindness Award and Foundation Fighting Blindness, Dennis W Jahnigen Award of the American Geriatrics Society, Joel Hoffman Scholarship, Crowley and Barbara \& Donald Jonas Family Funds. We thank Canon Inc., Tokyo, Japan for their technical support with the adaptive optics scanning laser ophthalmoscopy. Further gratitude is extended to the Dr Marcela Marsiglia for the referral of their patients for this study.

1 Fishman GA, Weinberg AB, McMahon TT: X-linked recessive retinitis pigmentosa: clinical characteristics of carriers. Arch Ophthalmol 1986; 104: 1329-1335.

2 Grover S, Fishman GA, Anderson RJ et al: A longitudinal study of visual function in carriers of X-linked recessive retinitis pigmentosa. Ophthalmology 2000; 107: 386-396.

3 Fishman GA, Cunha-Vaz JE: Carriers of $X$-linked recessive retinitis pigmentosa: investigation by vitreous fluorophotometry. Int Ophthalmol 1981; 4: 37-44.

4 Berson EL, Rosen JB, Simonoff EA: Electroretinographic testing as an aid in detection of carriers of X-chromosome-linked retinitis pigmentosa. Am J Ophthalmol 1979; 87: 460-468.

5 Arden GB, Carter RM, Hogg CR et al: A modified ERG technique and the results obtained in X-linked retinitis pigmentosa. Br J Ophthalmol 1983; 67: 419-430.

6 Merin S, Maggiano JM, Fishman GA: Retinal sensitivity under photopic conditions in patients with retinitis pigmentosa and carriers of the X-linked recessive form. Metabolic Ophthalmol 1978; 2: 225-229.

7 Ernst W, Clover G, Faulkner DJ: X-linked retinitis pigmentosa: reduced rod flicker sensitivity in heterozygous females. Invest Ophthalmol Vis Sci 1981; 20: 812-816.

8 Wegscheider E, Preising MN, Lorenz B: Fundus autofluorescence in carriers of X-linked recessive retinitis pigmentosa associated with mutations in RPGR, and correlation with electrophysiological and psychophysical data. Graefes Arch Clin Exp Ophthalmol 2004; 242: 501-511.

9 Vajaranant TS, Seiple W, Szlyk JP et al: Detection using the multifocal electroretinogram of mosaic retinal dysfunction in carriers of X-linked retinitis pigmentosa. Ophthalmology 2002; 109: 560-568.

10 Genead MA, Fishman GA, Lindeman M: Structural and functional characteristics in carriers of X-linked retinitis pigmentosa with a tapetal-like reflex. Retina 2010; 30: $1726-1733$.

11 Vingolo EM, Livani ML, Domanico D et al: Optical coherence tomography and electrooculogram abnormalities in X-linked retinitis pigmentosa. Doc Ophthalmol 2006; 113 : 5-10.

12 Fishman GA: Retinitis pigmentosa: genetic percentages. Arch Ophthalmol 1978; 96 : 822-826.

13 Bird AC: X-linked retinitis pigmentosa. Br J Ophthalmol 1975; 59: 177-199. 
14 Haim M: Retinitis pigmentosa: problems associated with genetic classification. Clin Genet 1993; 44: 62-70.

15 Berson EL, Gouras P, Gunkel RD et al: Dominant retinitis pigmentosa with reduced penetrance. Arch Ophthalmol 1969; 81: 226-234.

16 Ferreira PA: Insights into X-linked retinitis pigmentosa type 3, allied diseases and underlying pathomechanisms. Hum Mol Genet 2005; 2: R259-R267.

17 Neidhardt J, Glaus E, Lorenz B et al: Identification of novel mutations in X-linked retinitis pigmentosa families and implications for diagnostic testing. Mol Vis 2008; 6: 1081-1093.

18 Hosch J, Lorenz B, Stieger K: RPGR: role in the photoreceptor cilium, human retinal disease, and gene therapy. Ophthalmic Genet 2011; 32: 1-11.

19 Liang J, Williams DR: Aberrations and retinal image quality of the normal human eye. J Opt Soc Am A Opt Image Sci Vis 1997; 14: 2873-2883.

20 Liang J, Williams DR, Miller DT: Supernormal vision and high-resolution retinal imaging through adaptive optics. J Opt Soc Am A Opt Image Sci Vis 1997; 14: 2884-2892.

21 Roorda A, Romero-Borja F, Donnelly lii W, Queener H, Hebert T, Campbell M: Adaptive optics scanning laser ophthalmoscopy. Opt Express 2002; 10: 405-412.

22 Zhang Y, Poonja S, Roorda A: MEMS-based adaptive optics scanning laser ophthalmoscopy. Opt Lett 2006; 31: 1268-1270.

23 Zhang Y, Roorda A: Evaluating the lateral resolution of the adaptive optics scanning laser ophthalmoscope. J Biomed Opt 2006; 11: 014002.

24 Merino D, Duncan JL, Tiruveedhula P, Roorda A: Observation of cone and rod photoreceptors in normal subjects and patients using a new generation adaptive optics scanning laser ophthalmoscope. Biomed Opt Express 2011; 2: 2189-2201

25 Duncan JL, Zhang Y, Gandhi J et al: High-resolution imaging with adaptive optics in patients with inherited retinal degeneration. Invest Ophthalmol Vis Sci 2007; 48: 3283-3291.

26 Hirose F, Nozato K, Ken-ichi S et al: A compact adaptive optics scanning laser ophthalmoscope with high-efficiency wavefront correction using dual liquid crystal on silicon spatial light modulator. Proc SPIE 2011; 7885: 788515-1-788515-7.

27 Holder GE, Brigell MG, Hawlina M et al: ISCEV standard for clinical pattern electroretinography -2007 update. Doc Ophthalmol 2007; 114: 111-116.

28 Prokisch H, Hartig M, Hellinger R et al: A population-based epidemiological and genetic study of X-linked retinitis pigmentosa. Invest Ophthalmol Vis Sci 2007; 48 4012-4018.
29 Song $\mathrm{H}$, Chui TY, Zhong $\mathrm{Z}$ et al: Variation of cone photoreceptor packing density with retinal eccentricity and age. Invest Ophthalmol Vis Sci 2011; 52: 7376-7384.

30 Chui TY, Song H, Burns SA: Individual variations in human cone photoreceptor packing density: variations with refractive error. Invest Ophthalmol Vis Sci 2008; 49: 4679-4687.

31 Bech-Hansen NT, Pearce WG: X-linked retinitis pigmentosa: re-evaluation of fundus findings and the use of haplotype analysis in clarification of carrier female status. Ophthalmic Genet 1995; 16: 113-118.

32 Szamier RB, Berson EL: Retinal histopathology of a carrier of X-chromosome-linked retinitis pigmentosa. Ophthalmology 1985; 92: 271-278.

33 Adamian M, Pawlyk BS, Hong DH et al: Rod and cone opsin mislocalization in an autopsy eye from a carrier of X-linked retinitis pigmentosa with a Gly436Asp mutation in the RPGR gene. Am J Ophthalmol 2006; 142: 515-518.

34 Aguirre GD, Yashar BM, John SK et al: Retinal histopathology of an XLRP carrier with a mutation in the RPGR exon ORF15. Exp Eye Res 2002; 75: 431-443.

35 Lyon MF: Gene action in the X-chromosome of the mouse (Mus musculus L.). Nature 1961; 22: 372-373.

36 Machin GA: Some causes of genotypic and phenotypic discordance in monozygotic twin pairs. Am J Med Genet 1996; 61: 216-228.

37 Sandberg MA, Rosner B, Weigel-DiFranco $\mathrm{C}$ et al: Disease course of patients with $\mathrm{X}$-linked retinitis pigmentosa due to RPGR gene mutations. Invest Ophthalmol Vis Sci 2007; 48: 1298-1304.

38 Sharon D, Bruns GA, McGee TL et al: X-linked retinitis pigmentosa: mutation spectrum of the RPGR and RP2 genes and correlation with visual function. Invest Ophthalmol Vis Sci 2000; 41: 2712-2721.

39 Ebenezer ND, Michaelides M, Jenkins SA et al: Identification of novel RPGR ORF15 mutations in X-linked progressive cone-rod dystrophy (XLCORD) families. Invest Ophthalmol Vis Sci 2005; 46: 1891-1898.

40 Jalkanen R, Demirci FY, Tyynismaa $\mathrm{H}$ et al: A new genetic locus for $\mathrm{X}$ linked progressive cone-rod dystrophy. J Med Genet 2003; 40: 418-423.

41 Yang Z, Peachey NS, Moshfeghi DM et al: Mutations in the RPGR gene cause X-linked cone dystrophy. Hum Mol Genet 2002; 11: 605-611.

42 Brunner S, Skosyrski S, Kirschner-Schwabe R et al: Cone versus rod disease in a mutant rpgr mouse caused by different genetic backgrounds. Invest Ophthalmol Vis Sci 2010; 51: 1106-1115.

43 Thompson DA, Khan NW, Othman MI et al: Rd9 is a naturally occurring mouse model of a common form of retinitis pigmentosa caused by mutations in RPGR-ORF15. PLoS One 2012; 7: e35865. 